\title{
Yucca aloifolia seed oil: a new source of bioactive compounds
}

\begin{abstract}
Yucca aloifolia Linn (Y. aloifolia), also known as Spanish bayonet, is a drought-tolerant plant containing important bioactive compounds in various parts of the plant. Y. aloifolia is used as a natural medicinal herb. The purpose of the present study was to characterize and evaluate the seed oil extracted from Y. aloifolia seeds. The oil content of the seeds was $16.23 \%$. The principal fatty acids in the oil were linoleic acid (73.38\%), oleic acid (13.52\%) and palmitic acid $(8.18 \%)$. The oil has high vitamin $\mathrm{E}$ activity because of an appreciable concentration of tocols $(204 \mathrm{mg} / 100 \mathrm{~g})$, particularly tocotrienols, which represent $79 \%$ of the total amount of tocols. Tocotrienols have powerful antioxidant, anticancer, neuro/cardio protective and cholesterol-lowering properties. The thermal profile of Y. aloifolia seed oil was examined differential scanning calorimetry (DSC). Y. aloifolia seed oil is considered to be healthy dietary oil.
\end{abstract}

Keyword: Yucca aloifolia; Seed oil; Fatty acids; Tocotrienols; DSC; Health benefits 